\title{
Apicoectomies of Permanent Maxillary Incisors in Young Children with Different Retrograde Fillings: A Case Series
}

\author{
Vipin Ahuja ${ }^{1}$, Jaya Verma ${ }^{2}$, Annapurna Ahuja ${ }^{3}$
}

\begin{abstract}
Apicoectomy in children is considered as an anticipated treatment option to salvage a tooth with periapical pathology unresolved by conservative root canal treatment protocol. This methodology is a multidisciplinary systematic approach which includes endodontic procedures like root canal treatment, oral surgical procedures like resection of root apex, cavity preparation of root apex, and retrograde restoration of the root apex to achieve a bacteria-tight seal of the root canal system. This paper presents case series of 3 cases of children with complicated crown fractures of anterior teeth with periapical pathologies treated with apicoectomy and root end treatment with three different retrograde materials. The retrograde restorative approaches chosen were composite with bone grafting in Case 1, MTA in Case 2 and amalgam with bone grafting along with GTR membrane in Case 3 respectively. All the three cases showed successful treatment outcome during follow-up visits and presented with clinical and radiographic signs of eventful healing.

Keywords: Apical surgery, Apicoectomy, Pediatric endodontics, Retrograde filling, Root-end resection.

Journal of South Asian Association of Pediatric Dentistry (2021): 10.5005/jp-journals-10077-3095
\end{abstract}

\section{INTRODUCTION}

Traumatic injuries to oral tissues is one of the communal forms of injuries associated with pain, physical and psychological influences.' Dental traumatic injuries occur in school-going children commonly afflicting anterior region of mouth. ${ }^{2}$ Tooth injury is concomitant with the interruption of pulpal blood circulation leading to nonvitality of the pulp which in future may develop into endodontic infection. ${ }^{3}$

There are two endodontic ways to treat teeth with pulpal necrosis and periapical pathologies; the conservative way with root canal treatment and the radical way with periradicular surgery. In pathological conditions such as long standing periapical abscess, non-surgical root canal treatment with obturation and consequent coronal restoration eliminate detrimental pathogens. However, few cases do not heal even after the conventional endodontic treatment, and some even flare up, signifying the tenacity of potential microorganisms within the physical precincts of the canal system. In such conditions, surgery like apicoectomy become the favorable and established treatment option as documented in the literature for salvaging the affected tooth. ${ }^{4}$

Three imperative steps of periradicular surgery includes surgical debridement of pathological periradicular tissue, root-end resection (apicoectomy), and retrograde restoration. 5 "Root-resection", also known as apicoectomy was originated as a terminology for the amputation of the apices of roots in pulpless teeth as a protocol for treating dentoalveolar abscess in early eighties and has evolved as the chosen treatment option since then. ${ }^{6}$

Our case series of 3 cases present the successful treatment of trauma-induced periapical lesions of maxillary permanent incisors with open apices by apicoectomy with three different retrograde fillings.

\section{Case Descriptions}

\section{Case 1}

A male child of 11 years reported to the outpatient department of pediatric and preventive dentistry with the chief complaint of
${ }^{1,2}$ Pediatric and Preventive Dentistry, Hazaribag College of Dental
Sciences, Demotand, Hazaribagh, Jharkhand, India
${ }^{3}$ Periodontics and Implantology, Hazaribag College of Dental Sciences ${ }^{3}$ Periodontics and Implantology, Hazaribag
and Hospital, Hazaribagh, Jharkhand, India

Corresponding Author: Vipin Ahuja, Hazaribag College of Dental Sciences, Pediatric \& Preventive Dentistry, Demotand, Hazaribagh, Jharkhand, India, Phone: +91 9910178102, e-mail: drvipinahuja@gmail.com

How to cite this article: Ahuja V, Verma J, Ahuja A. Apicoectomies of Permanent Maxillary Incisors in Young Children with Different Retrograde Fillings: A Case Series. J South Asian Assoc Pediatr Dent 2021;4(3):203-207.

Source of support: Nil

Conflict of interest: None

pain and sensitivity in the right upper front region of the mouth since last month. The past medical history was not relevant. Previous dental history deciphered trauma to the upper anterior region of mouth 3 years ago, when the child was 8 years old. On clinical examination, the upper right lateral incisor presented with fractured crown, intraoral swelling and discoloration suggestive of complicated crown fracture traumatic injury with pulp necrosis (Fig. 1A). The tooth 12 was also tender on vertical percussion. Radiographic examination revealed periapical radiolucency with a diameter of $8 \times 8 \mathrm{~mm}$ in relation to 12 with open apex (Fig. 1B). As tooth no.12 was an immature tooth when it was traumatized, the root apex remained open. After radiographic and clinical assessment, a provisional diagnosis of periapical abscess was made. Varied treatment possibilities were elaborated to the patient such as closing apex with calcium hydroxide and other apexification agents; but as the patient was from a far place and wanted the procedure to be completed at the earliest, so we opted for periradicular surgical procedure. The written informed consent was taken and the surgical process was well explained to the patient.

\footnotetext{
(c) The Author(s). 2021 Open Access This article is distributed under the terms of the Creative Commons Attribution 4.0 International License (https://creativecommons. org/licenses/by-nc/4.0/), which permits unrestricted use, distribution, and non-commercial reproduction in any medium, provided you give appropriate credit to the original author(s) and the source, provide a link to the Creative Commons license, and indicate if changes were made. The Creative Commons Public Domain Dedication waiver (http://creativecommons.org/publicdomain/zero/1.0/) applies to the data made available in this article, unless otherwise stated.
} 


\section{Surgical procedure}

Blood investigations were carried to rule out any blood pathology. On the scheduled day of surgery, tooth no. 12 was first endodonically treated and over obturated with gutta percha. The overextended gutta-percha served as an identification marker for root end resection procedure. Local anesthesia was achieved by administering $2 \%$ lindocaine as infiltrations. Two vertical incisions and a cervicular incision were made to raise a full thickness mucoperiosteal flap (Fig. 1C). The trapezoidal flap was raised (Fig. 1D) and the bone defect was identified; the site for apicoectomy was confirmed with the help of an IOPA with a probe placed at the bone defect near the root of 12 (Fig. 1E). A window of more than $1 \times 1 \mathrm{~cm}$ diameter was created using straight micromotor handpiece with bone cutting burs under copious water spray (Fig. 1F). Root resection of up to $3 \mathrm{~mm}$ was done. Removal of granulation tissue was done using curettes and the excised tissue was fixed in $10 \%$ formalin for histopathological examination. The retrograde cavity was prepared with a contra-angle handpiece using a tapered fissure bur placing it at 45 degree angle from lingual to labial surface of the root (Fig. 1G). The cavity was then etched carefully by an etchant (Fig. 1H). Bonding agent was applied and light cured after which composite (Te-Econom Plus Composite Kit, Ivoclar-Vivadent, Liechtenstein) was cured to seal the apex (Fig. 11). Postoperative IOPA was taken to confirm the removal of extruded gutta-percha and root end restoration (Fig. 1J). The defect was large so bone graft (Advanced Biotech Osseograft DMBM, Chennai, India) was placed (Fig. 1K) and then after repositioning the flap, sutures were placed (Fig. 1L). The patient was recalled for suture removal after 10 days. Patient was reviewed after 1 and 3 months and was asymptomatic (Fig. $1 \mathrm{M})$. The histopathological biopsy report confirms periapical granuloma with 12 (Fig. 10).

\section{Case 2}

A 12 years old female patient came to the outpatient department of pediatric and preventive dentistry with a complaint of pain and swelling in the upper front region of the mouth since last week. The past medical history was not relevant. On recording past dental history, patient gave the history of trauma in the anterior region due to fall from bicycle 2 years ago. Clinical examination revealed tenderness on vertical percussion with moderate mobility with the right maxillary central incisor. On pulp vitality testing, tooth no.11 was inferred non vital. Radiographic examination revealed periapical radiolucency of $5 \times 5 \mathrm{~mm}$ with maxillary right central incisor. After radiographic and clinical assessment, a provisional diagnosis of periapical abscess was made.

\section{Surgical procedure}

Conventional endodontic treatment using calcium hydroxide was done as the first line of treatment for a year but as the patient was symptomatic, it was decided to go for the surgical apicoectomy procedure. Tooth no. 11 was endodonically treated and over obturated with gutta-percha as it served as an identification marker for root end resection. Local anesthesia was achieved by administering $2 \%$ lindocaine as infiltrations. Two vertical incision and a cervicular incision were given to raise a full thickness mucoperiosteal flap (Fig. 2A). The trapezoidal flap was raised and bone defect was identified (Fig. 2B). Apicoectomy site was
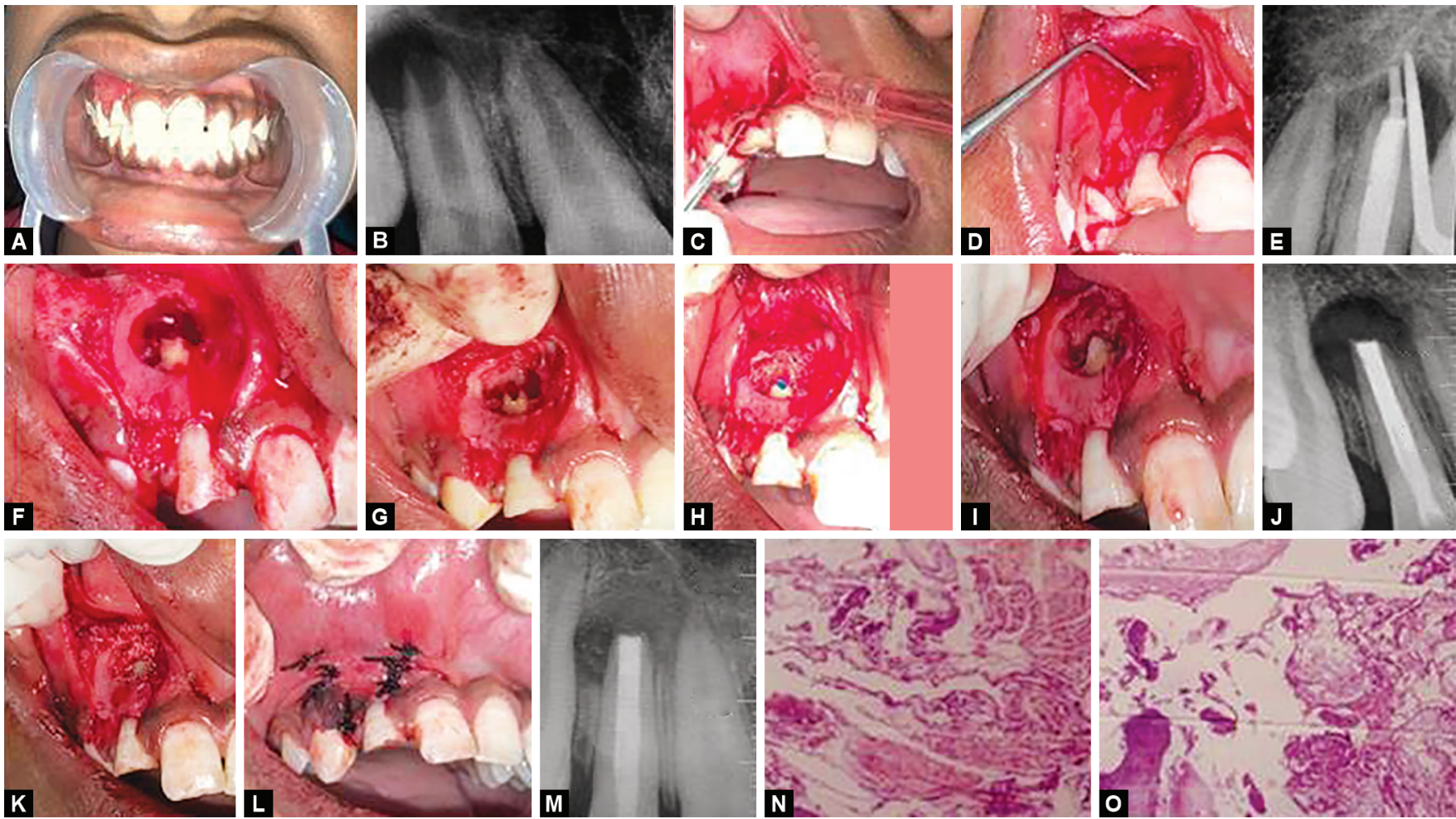

Figs 1A to O: A. Preoperative clinical picture of 12; B. Preoperative IOPA revealing 12 with open apex and periapical radiolucency; C. Surgical incision for quadrilateral flap, D. Flap raised and the bone defect detected, E. IOPA to confirm the site for apicoectomy, F. Round window of 1x1 $\mathrm{cm}$ created, G. Retrograde cavity prepared, H. Isolation, I. Retrograde composite restoration, J. Postoperative IOPA, K. Bone graft placed, L. Flap repositioned, M. Follow-up IOPA, N and O. Histopathological pictures 
confirmed with the help of an IOPA with a probe placed at the bone defect near the root of 11 (Fig. $2 \mathrm{H}$ ). A window of more than $6 \times 6 \mathrm{~mm}$ diameter was created using straight micromotor handpiece with bone cutting burs under copious water spray (Fig. 2C). Root resection of up to $3 \mathrm{~mm}$ was done. Removal of granulation tissue was done using curettes and the excised granulation material was sent for histopathological examination (Fig. 2D). The retrograde cavity was prepared with a contra-angle handpiece at 45 degree angle from lingual to labial surface of the root. In this case, the retrograde cavity was filled with MTA (Angelus, Brazil) and flap was repositioned and sutured without bone graft (Figs $2 \mathrm{E}$ and F). The histopathological biopsy report revealed non-specific periapical inflammation with 11 . The patient was reviewed after 1 and 3 months and was asymptomatic.

\section{Case 3}

An 11 years old female came to the outpatient department of pediatric and preventive dentistry with a complaint of pain and swelling in the upper left front region of mouth since two weeks (Fig.3A). Proper medical history was recorded and patient gave history of treated tuberculosis of lungs 6 months back. Past dental history revealed trauma to the anterior tooth region 1 year ago. On clinical examination, moderate sensitivity to percussion was present and the maxillary left central incisor was discolored and fractured. Preoperative radiograph and OPG were taken. The radiographic examination revealed periapical radiolucency of $7 \times 7 \mathrm{~mm}$ in diameter with left central incisor which was endodontically treated previously. After case history, radiographic and clinical assessment, a provisional diagnosis of previous root canal treatment with symptomatic apical periodontitis with 21 was made. The written informed consent was taken and the surgical process was correctly explained to the patient.

\section{Surgical procedure}

Local anesthesia was achieved by administering $2 \%$ lindocaine as infiltrations. Two vertical incision and a cervicular incision were given to raise a full thickness mucoperiosteal flap (Fig. 3B). The trapezoidal flap was raised and bone defect was identified (Fig. 3C). Bony window of $1 \times 1 \mathrm{~cm}$ was made using straight micromotor handpiece with bone cutting burs under copious water spray (Fig.
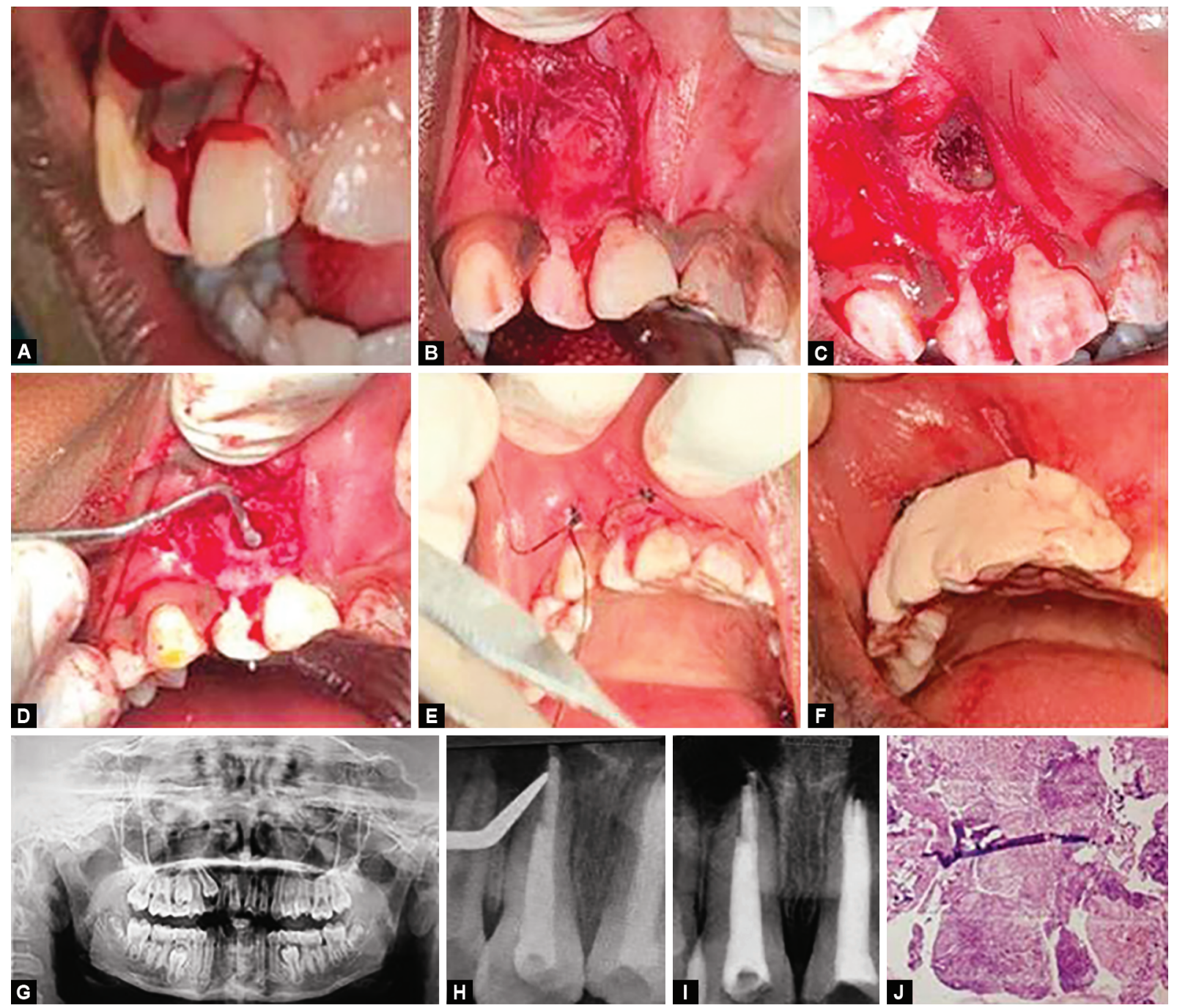

Figs $2 \mathrm{~A}$ to J: A. Surgical incisions given, B. Full-thickness mucoperiosteal flap raised and bone defect detected, C. Surgical window created in bone and extra gutta-percha seen, D. Retrograde cavity prepared and filled with MTA, E. Flap repositioned and sutured, F. Surgical COE-Pack given, G. Pre-operative OPG, H. IOPA with diagnostic probe through the bone defect to confirm the site for apicoectomy, I. Postoperative IOPA, J. Histopathological section suggestive of non-specific inflammation 
3D). Root of tooth no. 21 was resected up to $3 \mathrm{~mm}$. The retrograde cavity was prepared with a contra-angle hand piece at $45^{\circ}$ angle from lingual to labial surface of the root (Fig. 3E). Removal of granulation tissue was done using curettes and the excised specimen was sent for histopathological examination (Fig. 3E). Retrograde cavity was filled with amalgam (DPI, Mumbai, India) and bone graft (Advanced Biotech Osseograft DMBM, Chennai, India) was packed due to the large size of bone defect (Fig. 3F). In addition to this, GTR membrane (Advanced Biotech Healiguide, Chennai, India) was placed over the cavity with filled bone graft to aid in stabilization of the graft and eventful healing (Fig. 3G). The raised flap was sutured back and hisopathological report confirmed periapical granuloma with 21. Patient was reviewed at 1, 3 and 6 month post-surgery and was asymptomatic.

\section{Discussion}

Apical surgery is an invasive treatment approach used in cases not responding to conservative endodontic approach and is a standardized surgical treatment method. The rationale is to save the infected tooth with a persistent periapical lesion and preserve it in the dental arch by surgically eliminating the bacterial load surrounding the root area and packing it with a retrograde restoration following root-end resection procedure. Apical surgery methods include periapical curettage with planing, apicoectomy, apicoectomy with retro-filling, apicoectomy with retro-instrumentation and filling and filling concomitant to surgery. ${ }^{7,8}$

The success of apicoectomy is dependent on diverse factors and is corroborated during follow up through clinical and radiographic evaluations. As put into view by Weine, indications for apical surgery are as follows: ${ }^{9}$

1. Severe vertical bone loss encompassing only one root of multi-rooted teeth.

2. Through and through furcation involvement.

3. Unfavorable closeness of roots of adjacent teeth.

4. Precluding adequate hygiene maintenance in proximal areas.

5. Severe root exposure due to dehiscence.

There is a shift in paradigm in retrograde filling materials from amalgam, glass ionomer cement and composites to MTA and SuperEBA or IRM nowadays. ${ }^{10,11}$ In our case series, endodontic management of traumatized maxillary incisors with open apex of three children using different retrograde restorations is described. The different retrograde materials used as retrograde cavity fillings were composite, MTA and amalgam respectively. Mineral trioxide aggregate is known as a successful root-end filling material because of its exceptional sealing capability and ability to stimulate osteoblast activity. ${ }^{12}$ In addendum to these, MTA is antimicrobial and
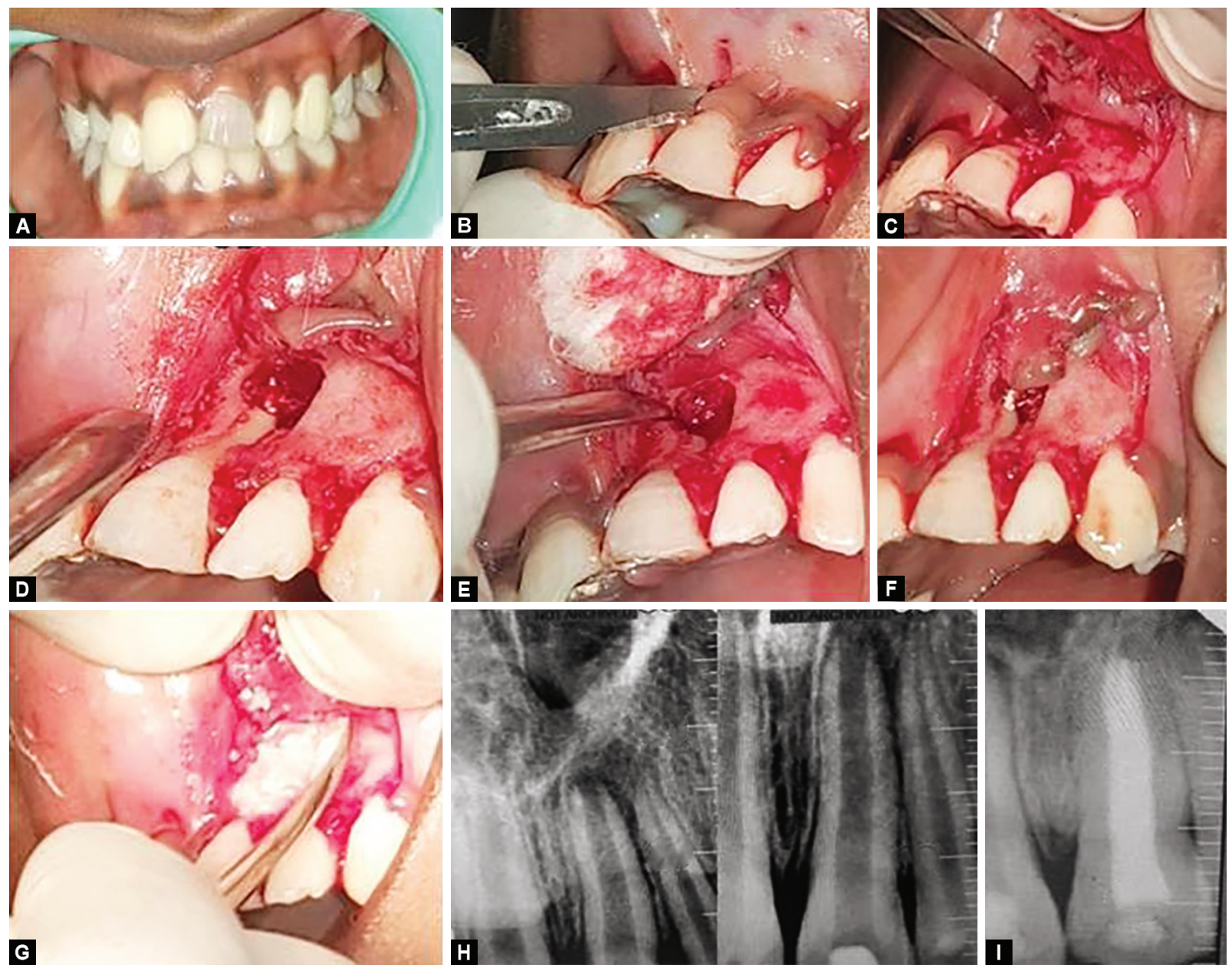

Figs 3A to I: A. Pre-operative clinical presentation of 21, B. Surgical incisions given, C. Full-thickness mucoperiosteal flap raised and bone defect detected, D. Bone window prepared and over extended gutta-percha seen, E. Granulation tissue removed and cavity is cleaned and irrigated, F. Bone graft placed, G. GTR membrane placed in the cavity, H. Pre-operative IOPA, I. Post-operative IOPA 
also showed least cytotoxic effects when compared to amalgam, IRM, or SuperEBA. Previous animal studies on MTA had established the medicament as least inflammatory with potential cementogenic property with cementum bridge formations directly over the MTA root-end fillings. ${ }^{13}$

Guided tissue regeneration (GTR) techniques have also been proposed as an adjunct to periapical healing post-surgery but are sparsely used in pediatric surgical endodontic procedures. In our third case, GTR membrane was used over the packed bone graft to stabilize the bone graft and enhance healing process as the patient had a past medical history of compromised immunity because of tuberculosis of lungs. ${ }^{14}$ Published case of peri-radicular surgery using amalgamation of MTA, bovine bone graft and cortical collagen membrane acclaimed pronounced success. It was concluded that technique is favorable for osseous healing and can be a savior for tooth with hopeless prognosis. It aids in periodontal structures regeneration after periapical surgery. ${ }^{15}$

In spite of using three different retrograde systems of restorations with varied bone augmentation techniques, all the cases showed successful outcome.

\section{Conclusion}

- Apicoectomy is the standardized radical pulp therapy procedure used where periapical lesions do not ebb with conservative root canal procedures.

- Retrograde restorations like composite, MTA and amalgam are equivalently effective as retro-sealing materials.

- Large bony defects can be effectually augmented by osseografts with or without GTR membranes.

\section{Important Learning Points}

- Apicoectomy is an invasive procedure but very effective with outstanding prognosis if case selection is properly done.

- In our case series, composite was used as retrograde filling material in Case 1, which was technique sensitive and difficult to restore to a satisfactory level as isolation could not be ensured at the site of surgery. Hence, we advise to go for MTA or Amalgam over Composite for retrograde restorations in apicoectomy procedures.

- In Case 3, GTR membrane was used to stabilize the bone graft which is effectively used for this purpose before as documented in literature. But, no clinically significant differences in treatment outcome was inferred in between Case 1 without GTR membrane and Case 3 with GTR membrane. However, large sample studies of longitudinal protocol are recommended to ratify this finding.

\section{References}

1. Berman LH, Blanco L, Cohen S. A clinical guide to dental traumatology. USA: Elsevier Health Sci; 2006: Available from: https://www.elsevier.com/books/a-clinical-guide-to-dentaltraumatology/9780323040396.

2. Skaare $A B$, Jacobsen I. Dental injuries in Norwegians aged 7-18 years: dental injuries in young Norwegians. Dent Traumatol 2003;19(2):6771. DOI: 10.1034/j.1600-9657.2003.00133.x

3. Garlapati R, Venigalla BS, Patil JD, et al. Surgical management of mandibular central incisors with dumbbell shaped Periapical Lesion: a case report. Case Rep Dent 2014:1-5. DOI: 10.1155/2014/769381

4. Von Arx T, Walker WA. Microsurgical instruments for root-end cavity preparation following apicoectomy: a literature review: retrotips in apicoectomy. Dent Traumatol 2000;16(2):47-62. DOI: 10.1034/j.16009657.2000.016002047.x

5. Reit C, Hirsch J. Surgical endodontic retreatment. Int Endod J 1986;19:107-112. DOI: 10.1111/j.1365-2591.1986.tb00462.x

6. Seldin SD. Apicectomy. Am J Orthod 1947;33(4):301-308. DOI: 10.1016/0096-6347(47)90066-5

7. Quality guidelines for endodontic treatment: consensus report of the European Society of Endodontology. Int Endod J 2006;39(12):921-930. DOI: $10.1111 / j .1365-2591.2006 .01180 . x$

8. Avinash S, Agrawal E, Mushtaq I, et al. Apicoectomy: an elucidation to a hitch. J Dent Specialities 2019;7(1):28-32. DOI: 10.18231/j. jds.2019.006

9. Weine FS. Endodontic therapy, 6th edition; Mosby Inc.2004:423-51.

10. Zuolo ML, Ferreira MOF, Gutmann JL. Prognosis in periradicular surgery: a clinical prospective study. Int Endod J 2000;33(2):91-98. DOI: 10.1046/j.1365-2591.2000.00263.x

11. Johnson BR: Considerations in the selection of a root-end filling material. Oral Surg Oral Med Oral Pathol Oral Radiol Endod 1999; 87: 398-404. DOI: 10.1016/s1079-2104(99)70237-4

12. Torabinejad M, Pitt Ford TR: Root-end filling material: a review. Endod Dent Traumatol 1996;12:161-178. DOI: 10.1111/j.1600-9657.1996. tb00510.x

13. Iqbal $N$. A case report on apicoectomy followed by retrodgrade filling and perforation repair. Int J Med Sci Diagn Res 2020;4(6):27-29.

14. Tsesis I, Rosen E, Tamse A, et al. Effect of guided tissue regeneration on the outcome of surgical endodontic treatment: a systematic review and meta-analysis. J Endod 2011;37(8):1039-1045. DOI: 10.1016/j. joen.2011.05.016

15. Bernabé PF, Azuma MM, Ferreira LL, et al. Root reconstructed with mineral trioxide aggregate and guided tissue regeneration in apical surgery: a 5-year follow-up. Braz Dent J 2013;24(4):428-432. DOI: 10.1590/0103-6440201302242 